\title{
Pulmonary Infection Is an Independent Risk Factor for Long-Term Mortality and Quality of Life for Sepsis Patients
}

\author{
Xiao-Li He, Xue-Lian Liao, Zhi-Chao Xie, Li Han, Xiao-Lei Yang, and Yan Kang \\ Department of Critical Care Medicine, West China Hospital, Sichuan University, Chengdu, China \\ Correspondence should be addressed to Yan Kang; kang_yan_123@163.com
}

Received 14 July 2016; Revised 31 August 2016; Accepted 26 October 2016

Academic Editor: Ruxana Sadikot

Copyright ( 2016 Xiao-Li He et al. This is an open access article distributed under the Creative Commons Attribution License, which permits unrestricted use, distribution, and reproduction in any medium, provided the original work is properly cited.

\begin{abstract}
Background. Long-term outcomes (mortality and health-related quality of life) of sepsis have risen as important indicators for health care. Pulmonary infection and abdominal infection are the leading causes of sepsis. However, few researches about long-term outcomes focused on the origin of sepsis. Here we aim to study the clinical differences between pulmonary-sepsis and abdominalsepsis and to investigate whether different infection foci were associated with long-term outcomes. Methods. Patients who survived after hospital discharge were followed up by telephone interview. Quality of life (QoL) was assessed using the EuroQol 5-dimension (EQ5D) questionnaire. Results. Four hundred and eighty-three sepsis patients were included, 272 (56.3\%) had pulmonary-sepsis, and $180(37.3 \%)$ had abdominal-sepsis. The overall ICU and one-year mortality rates of the cohort were $17.8 \%$ and $36.1 \%$, respectively. Compared with abdominal-sepsis, pulmonary-sepsis patients had older age, higher APACHE II, higher ICU mortality (31.7\% versus $12.6 \%$ ), and one-year mortality ( $45.4 \%$ versus $24.4 \%$ ), together with worse QoL. Age, septic shock, acute renal failure, fungus infection, anion gap, and pulmonary infection were predictors for one-year mortality and pulmonary infection was a risk factor for poor QoL. Conclusions. Pulmonary-sepsis showed worse outcome than abdominal-sepsis. Pulmonary infection is a risk factor for one-year mortality and QoL after sepsis.
\end{abstract}

\section{Introduction}

Despite advances in organ support and guidelines for sepsis management, the incidence of sepsis is still increasing [15]. Sepsis is the leading cause of death among hospitalized patients [6], mortality of which ranging from 20 to $80 \%$. Sepsis survivors also suffered persistent reduction in long-term health-related quality of life (HR-QoL), such as depression, morbidity, and cognitive impairment [7-9]. This reduction can persist up to 5 years after hospital discharge [10]. For better evaluation of the long-term outcomes of sepsis, we should focus not only on its long-term mortality but also on HR-QoL.

More and more researches have showed that the EQ5D questionnaire can be used in critically ill patients to evaluate long-term HR-QoL [11-13]. The EQ5D questionnaire includes five dimensions, namely, mobility ability, self-care, usual activity, pain/discomfort, and anxiety/depression. Each dimension has three different levels, separately none, moderate, and severe problem. An EQ5D index can be obtained based on the EQ5D questionnaire via a Japanese version conversion table [14]. The visual analog scale (VAS), as a part of the questionnaire, is also used. The EQ-VAS, a score ranging from 0 to 100 , can subjectively reflect the health state of patients, where 0 means the worst state and 100 the best [14].

Pneumonia is one of the most common reasons for admission to intensive care units (ICUs). Studies have revealed that pneumonia is the primary kind of sepsis [1517]. Kim and his colleagues' study showed that pneumonia is associated with higher mortality when compared to other infection sources [18].

Abdominal infection is another common indication for admission to ICU, and abdomen is the second popular site of invasive infection among critically ill patients [19-21]. Poor control of abdominal infection frequently results in abdominal-sepsis [22].

Lung and abdomen are the most common sources of sepsis $[4,12]$. Existing research on the outcome of sepsis according to the infection foci is sparse and information about 
difference between pulmonary-sepsis and abdominal-sepsis is still limited. Our study focuses on elucidating the clinical difference between pulmonary-sepsis and abdominal-sepsis, the variation in long-term mortality, and QoL of different sepsis origin and identifying the predictors of long-term mortality and QoL for sepsis survivors.

\section{Materials and Methods}

2.1. Study Population. This prospective cohort study was carried out among patients admitted to the combined surgical, respiratory, and medical intensive care units of West China hospital of Sichuan University (from December 2013 to December 2014). Patients diagnosed with sepsis as the primary cause for ICU administration were identified and enrolled within the first 24 hours. Patients younger than 18 years were excluded, and so were patients with a length of ICU stay less than 24 hours. If the patient was admitted to the ICU more than once, only the first sepsis episode was enrolled. HR-QoL was assessed using the EQ5D questionnaire. Permission to perform the follow-up study was granted by the Clinical Trials and Biomedical Ethics Committee of West China Hospital.

2.2. Definitions, Data Collection, and Outcome Measures. Sepsis was defined as at least two systemic inflammatory syndrome criteria together with infection evidence [23]. At least one of the following criteria was required for diagnosis of pneumonia: (1) clinical features including fever $\left(>38^{\circ} \mathrm{C}\right)$ or hypothermia $\left(\leq 35^{\circ} \mathrm{C}\right)$, new cough wherever with or without sputum, dyspnea, pleuritic chest pain, or changed respiratory sounds; (2) radiographic evidence of lung infection with a newly onset or changed infiltrate focus based on the guidelines of German College of Pulmonology [24]. Abdominal infection includes bacterial liver abscess, acute peritonitis, acute binary tract infection cholecystitis, and acute pancreatitis complicated with secondary bacterial infections.

Demographic characteristics, infection site, type of infection (G+/G-, fungus, or virus), laboratory results in the first 24 hours, comorbidities, length of ICU and hospital stay, ICU administration strategy such as mechanical ventilation, continuous renal replacement therapy (CRRT), and use of vasoactive agent were recorded. The Acute Physiology and Chronic Health Evaluation (APACHE) II score [25] and Sepsis-related Organ Failure Assessment (SOFA) score [26] in the first 24 hours of ICU admission, were also collected to assess the severity of illness. Primary outcome was one-year mortality, and secondary outcome was one-year QoL assessed via EQ5D. All clinical data were obtained from the Hospital Information System of West China Hospital and follow-up information was recorded by the telephone interviewer.

2.3. Statistical Analysis. Statistical analysis was conducted in SPSS software version 19.0 (SPSS Inc., Chicago, IL, USA). The Kolmogorov-Smirnov test was used to assess the data normality. Quantitative data exhibiting normal distributions were expressed as mean and standard deviation (SD) or, otherwise, presented as median with 25 th and 75 th percentile on rejection of the normality hypothesis. Students' $t$-test was used for the analysis of normally distributed continuous variables. The Mann-Whitney test was used to explore the difference between the independent groups when the data was not normally distributed. For categorical variables, the $\chi 2$ (for large sample) or Fisher's exact test (for small sample) was applied appropriately to calculate the difference between groups. Backward stepwise binary logistic regression was conducted to find predictors for one-year mortality and QoL after the sepsis episode. All the tests were two-tailed and a $p$ value less than 0.05 was considered statistically significant. Missing data were handled via simple deletion method and patients lost to follow-up were excluded when analyzing oneyear mortality and quality of life.

\section{Results}

Study flow was presented in Figure 1. Of the 483 patients, 86 died in ICU and 11 died in ward. Three hundred and eighty-six hospital survivors were followed up by telephone one year after ICU discharge. Forty-eight patients were lost to follow-up. Of the others, 216 patients survived one year after ICU discharge, and then EQ5D questionnaire was used for the assessment of QoL for 1-year survivors and 209 of them finished the questionnaire (Figure 1).

3.1. Demographic Characteristics of the Sepsis Cohort. Among the 483 sepsis patients, pulmonary-sepsis $(56.3 \%, n=272)$ was the most common type of sepsis, followed by abdominal infection $(37.3 \%, n=180)$. The mean age of the sepsis cohort was 60.3 years, and the average APACHE II score was 21.5. For the whole study population, the incidence of sepsis, severe sepsis, and septic shock was $7.7 \%, 27.5 \%$, and $64.8 \%$, respectively. Pulmonary-sepsis patients were much older (63.7 years old) than abdominal-sepsis patients (56.7 years old) ( $p=$ 0.000 ) and had higher APACHE II score (23.0 versus 18.6, $p=0.000$ ). The SOFA score of pulmonary-sepsis (median 9, IQR 7-12) was significantly worse than that of abdominalsepsis (median 7, IQR 5-11). Pulmonary-sepsis had a higher Charlson Comorbidity Index. Fungal or viral infection was more likely to be identified in the pneumonia-induced sepsis population. Pulmonary-sepsis was more prone to develop acute renal failure $(17.6 \%, p=0.043)$ and had greater need for CRRT during the whole ICU stay period $(20.2 \%$ and $11.1 \%, p=0.014)$. Pulmonary-sepsis had longer MV days and length of ICU stay $(p=0.000)$. Demographic characteristics of the study cohort were presented in Table 1.

3.2. Mortality of the Sepsis Cohort. ICU mortality for all sepsis, pulmonary-sepsis, and abdominal-sepsis was $17.8 \%$ $(n=86), 22.4 \%(n=61)$, and $8.9 \%(n=16)$, respectively, and hospital mortality was $20.1 \%(n=97), 25.0 \%$ ( $n=68)$, and $10.0 \%(n=18)$, respectively (Table 1$)$. The overall 28-day mortality of the ICU survivors for all sepsis, pulmonary-sepsis, and abdominal-sepsis was $23.4 \%(n=79)$, $31.7 \%(n=58)$, and $12.6 \%(n=17)$, respectively, and oneyear mortality of ICU survivors was $36.1 \%(n=122), 45.4 \%$ $(n=83)$, and $24.4 \%(n=33)$, respectively (Table 1$)$ (when 


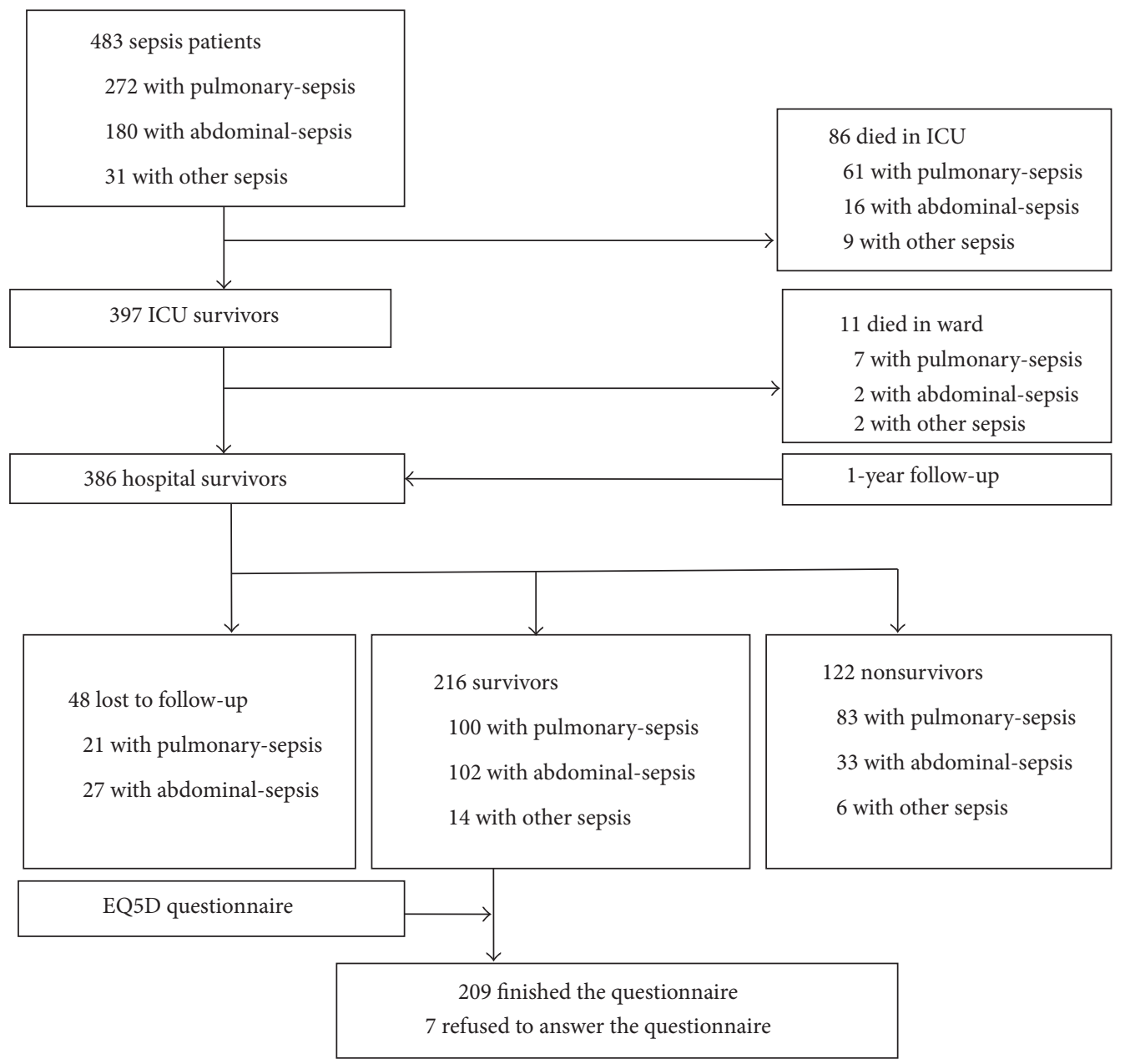

FIgUre 1: Flow chart of the follow-up study. EQ5D, EuroQol-5D.

analyzing one-year mortality, all sepsis $n=437$, patients lost to follow-up were excluded). Kaplan-Meier curve also showed that patients with pulmonary-sepsis had higher oneyear mortality than that of the patients with abdominalsepsis (Figure 2(a)). Considering the older age and greater comorbidity burden on the pulmonary-sepsis cohort, we did an age-matched cohort study of ICU survivors to adjust the impact on long-term mortality. Similar results were obtained; that is, pulmonary-sepsis showed poor survival (Figure 2(b)). Background characteristics of the age-matched cohort were shown in Supplementary Table 1 in Supplementary Material available online at http://dx.doi.org/10.1155/2016/4213712.

3.3. One-Year QoL (EQ5D) of Sepsis Survivors. The distribution of the five dimensions in the EQ5D questionnaire was described in Table 2. Of all the survivors who completed the EQ5D questionnaire, $18.7 \%$ had moderate to severe problem in mobility, $12.5 \%$ in self-care, $19.2 \%$ in pain/discomfort, $33.5 \%$ in anxiety/depression, and $19.1 \%$ in anxiety/depression. This showed that most patients had problems in the pain/discomfort dimension. The median EQ5D index was 0.848, and the median EQ-VAS was
80. Pulmonary-sepsis patients showed more problems than abdominal-sepsis patients in all the five dimensions (Figure 3, Table 2). Significant difference was found in both the EQ5D index and EQ-VAS ( $p=0.001$ for both). Pulmonary-sepsis patients showed worse one-year QoL (Table 2).

3.4. Risk Factors for One-Year Mortality. To find risk factors for one-year mortality, a total of 435 sepsis patients were involved in the analysis. Of them, 216 (49.7\%) survived one year after ICU discharge. Nonsurvivors tended to be much older and had apparently higher APACHE II, SOFA, and Charlson Comorbidity Index (all $p=0.000$ ). Greater ratio of patients in nonsurvivors was identified with fungal infection $(p=0.000)$. The incidence of septic shock was obviously higher in the nonsurvivor group $(74.8 \%)$ than that of the survivor group $(56.0 \%)(p=0.000)$. One hundred and fiftytwo of the $219(69.7 \%)$ nonsurvivors had pulmonary-sepsis, which was much higher than the survivor group (46.3\%). However, abdominal-sepsis was more frequently found in the survivor group $(47.2 \%$ versus $22.9 \%, p=0.000)$. Within the first 24 hours after admission to the ICU, there was a greater need of vasopressor use for the nonsurvivors $(47.2 \%$ 


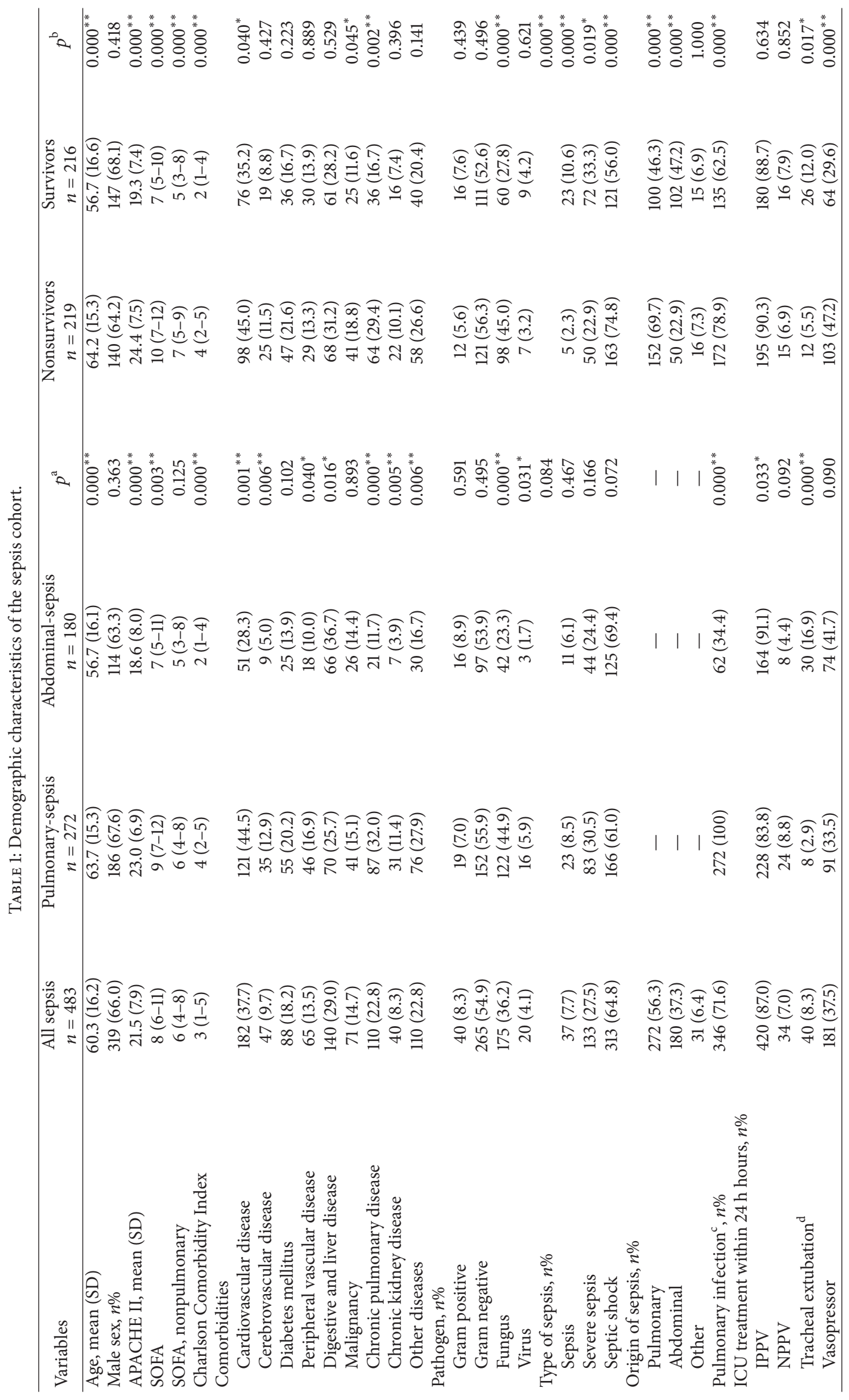




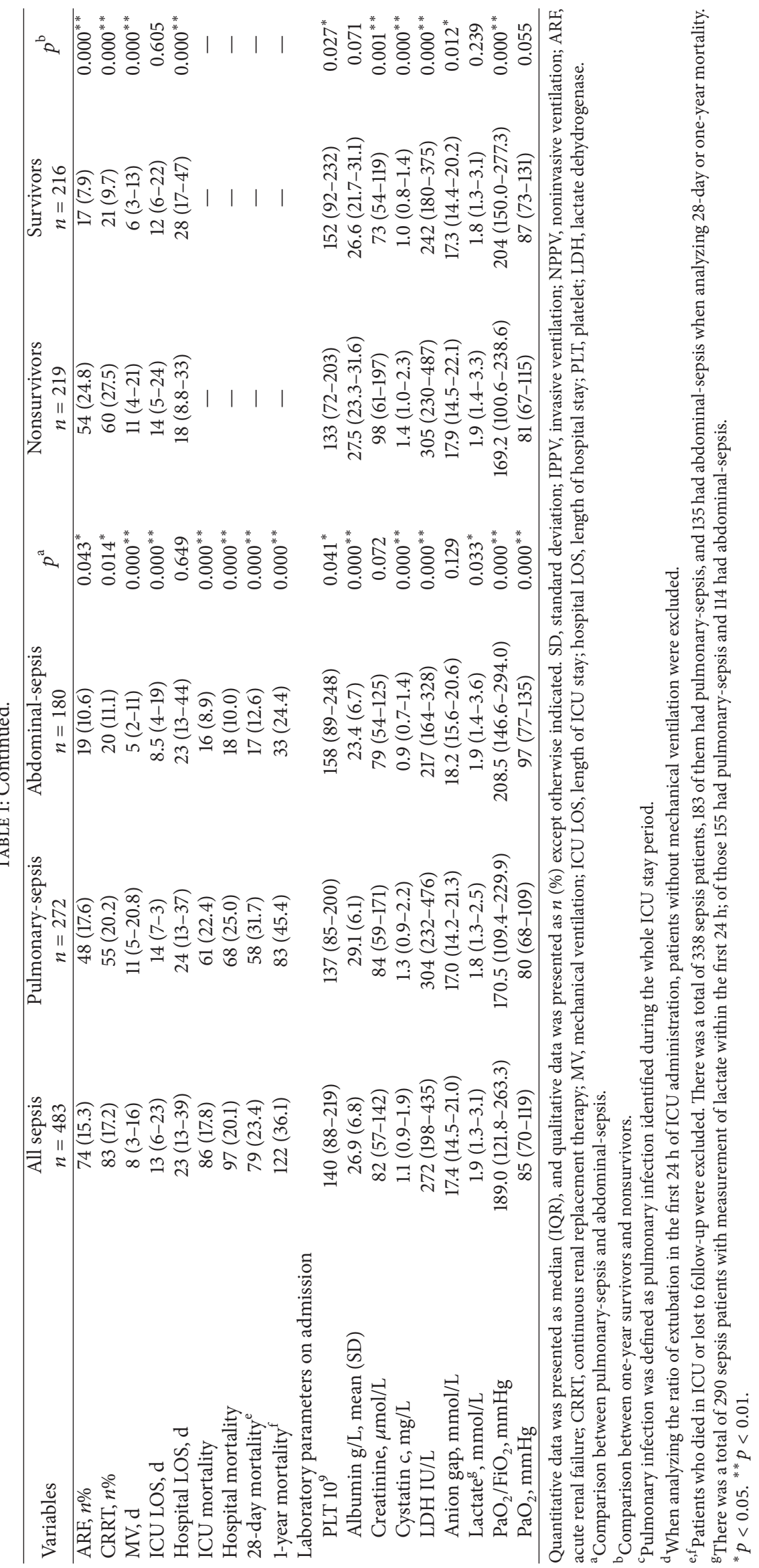




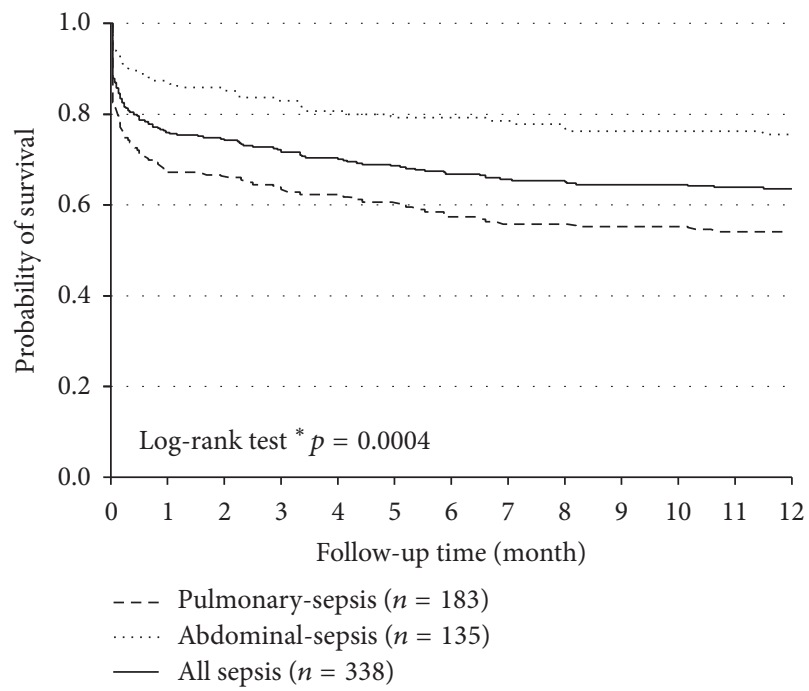

(a)

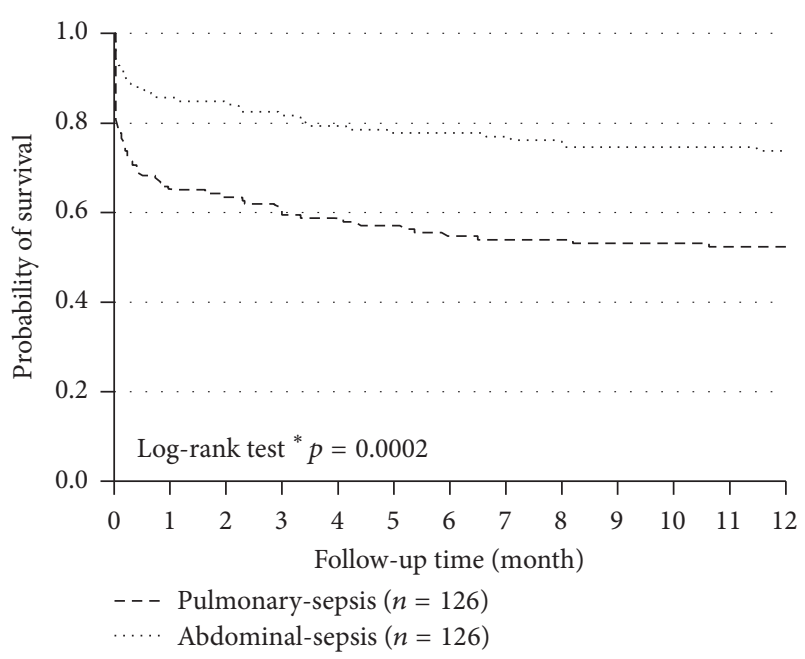

(b)

FIgURe 2: Probability of one-year survival for ICU survivors. (a) Probability of one-year survival for all ICU survivors of the unmatched cohorts. (b) Probability of one-year survival for ICU survivors of the age-matched cohorts. ${ }^{*} p$ value indicated for comparison between pulmonary-sepsis and abdominal-sepsis.

TABLE 2: One-year HR-QoL (EQ-5D) of sepsis survivors and comparison between pulmonary-sepsis and abdominal-sepsis.

\begin{tabular}{|c|c|c|c|c|}
\hline Variable & $\begin{array}{c}\text { Sepsis responders } \\
n=209\end{array}$ & $\begin{array}{c}\text { Pulmonary-sepsis } \\
n=97\end{array}$ & $\begin{array}{c}\text { Abdominal-sepsis } \\
n=98\end{array}$ & $p$ \\
\hline \multicolumn{5}{|l|}{ Mobility } \\
\hline No problems & $170(81.3)$ & $70(72.2)$ & $91(92.9)$ & $0.000^{* *}$ \\
\hline Some problems & $25(12.0)$ & $18(18.6)$ & $5(5.1)$ & $0.004^{* *}$ \\
\hline Extreme problems & $14(6.7)$ & $9(9.3)$ & $2(2.0)$ & $0.033^{*}$ \\
\hline \multicolumn{5}{|l|}{ Self-care } \\
\hline No problems & $183(87.6)$ & $78(80.4)$ & $95(96.9)$ & $0.000^{* *}$ \\
\hline Some problems & $10(4.8)$ & $8(8.2)$ & $1(1.0)$ & $0.018^{*}$ \\
\hline Extreme problems & $16(7.7)$ & $11(11.3)$ & $2(2.0)$ & $0.010^{*}$ \\
\hline \multicolumn{5}{|l|}{ Usual activity } \\
\hline No problems & $169(80.9)$ & $67(69.1)$ & $93(94.9)$ & $0.000^{* *}$ \\
\hline Some problems & $25(12.0)$ & $20(20.6)$ & $3(3.1)$ & $0.000^{* *}$ \\
\hline Extreme problems & $15(7.2)$ & $10(10.3)$ & $2(2.0)$ & $0.018^{*}$ \\
\hline \multicolumn{5}{|l|}{ Pain/discomfort } \\
\hline No problems & $139(66.5)$ & $56(57.7)$ & $72(73.5)$ & $0.024^{*}$ \\
\hline Some problems & $64(30.6)$ & $39(40.2)$ & $23(23.5)$ & $0.014^{*}$ \\
\hline Extreme problems & $6(2.9)$ & $2(2.1)$ & $3(3.1)$ & 0.505 \\
\hline \multicolumn{5}{|l|}{ Anxiety/depression } \\
\hline No problems & $169(80.9)$ & $72(74.2)$ & $86(87.8)$ & $0.018^{*}$ \\
\hline Some problems & $35(16.7)$ & $23(23.7)$ & $10(10.2)$ & $0.013^{*}$ \\
\hline Extreme problems & $5(2.4)$ & $2(2.1)$ & $2(2.0)$ & 1.000 \\
\hline EQ5D index (IQR) & $0.848(0.729-0.848)$ & $0.768(0.668-0.848)$ & $0.848(0.768-0.848)$ & $0.000^{* *}$ \\
\hline$E Q-V A S(I Q R)$ & $80(68.7-90)$ & $75(60-85)$ & $80(70-90)$ & $0.001^{* *}$ \\
\hline
\end{tabular}

Data was presented as $n$ (\%). Patients who refused to finish the questionnaire were excluded. ${ }^{*} p<0.05 .{ }^{* *} p<0.01$.

versus $29.6 \%, p=0.000)$. Similarly, nonsurvivors were more prone to develop acute renal failure than survivors and had more requirements for CRRT. Mechanical days and length of hospital stay $(p=0.000)$ were also longer in the nonsurvivors group, but there was no difference in ICU LOS $(p=0.605)$.
Laboratory parameters such as creatinine, plates, cystatin c, $\mathrm{LDH}$, and anion gag were also worse in the nonsurvivors (Table 1).

Univariate analysis of the mortality showed age, APACHE II, SOFA, Charlson Comorbidity Index, malignancy, acute 


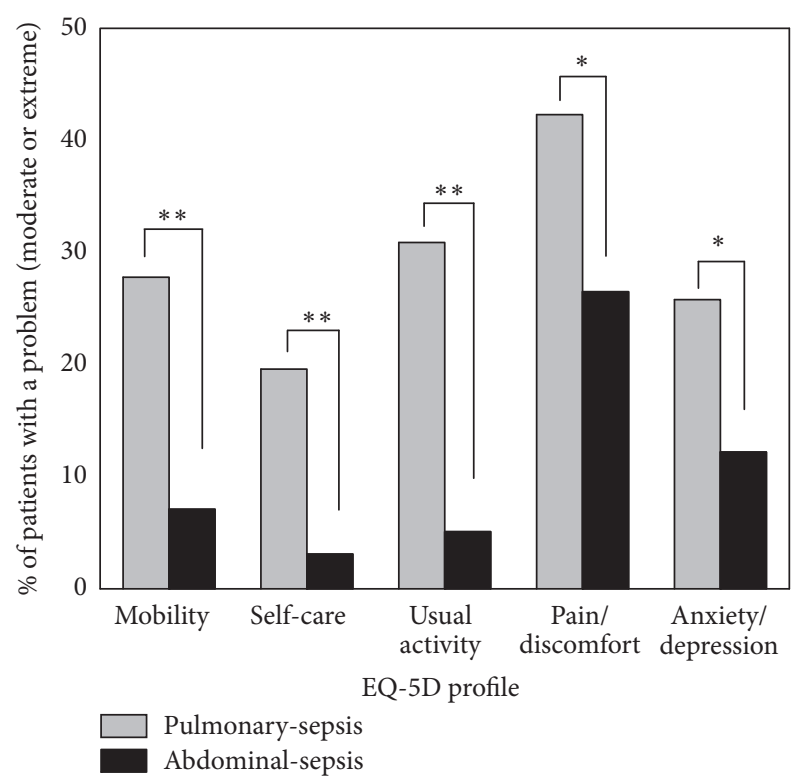

FIGURE 3: EQ5D profile in one-year survivors of pulmonary-sepsis and abdominal-sepsis. The EuroQol 5D profile is dichotomized into "no problems" and "moderate or extreme problems" 1 year after ICU discharge ${ }^{*} p<0.05 ;{ }^{* *} p<0.01$.

renal failure, pulmonary infection, fungus infection, septic shock, cystatin c, and anion gap as potential predictors for one-year mortality. After multivariate adjustment, age (OR $=1.025 ; 95 \%$ CI $1.011-1.039)$, septic shock $(\mathrm{OR}=2.533 ; 95 \%$ $\mathrm{CI}, 1.591-4.032)$, fungus infection $(\mathrm{OR}=1.846$; $95 \% \mathrm{CI}, 1.160$ 2.938), acute renal failure ( $\mathrm{OR}=2.914 ; 95 \% \mathrm{CI}, 1.525-5.568)$, anion gap ( $\mathrm{OR}=1.070$; $95 \% \mathrm{CI}, 1.025-1.117)$, and pulmonary infection $(\mathrm{OR}=2.547 ; 95 \% \mathrm{CI}, 1.513-4.288)$ were risk factors for one-year mortality (Table 3 ).

3.5. Predictors for One-Year Quality of Life. In order to find predictors for one-year QoL, QoL was evaluated by EQ5D index. EQ5D index less than 0.848 (median) was defined as poor QoL. Survivors were divided into poor and good QoL groups. Background characteristics were summarized in Table 4. Patients with poor QoL had higher APACHE II and Charlson Comorbidity Index, prolonged mechanical ventilation, longer ICU, and hospital LOS. Patients of the poor QoL group were more prone to suffer pulmonary infection $(76.7 \%$ versus $47.2 \%)$, and $57.3 \%$ of them had pulmonary-sepsis, while only $25.8 \%$ of patients in the good QoL group had pulmonary-sepsis. Univariate analysis suggested that APACHE II, chronic heart failure, pulmonary infection, and tube extubation during the first 24 hours after admission to ICU were possible predictive factors of one-year QoL (Table 3). Multivariate logistic regression showed that pulmonary infection $(\mathrm{OR}=2.846,95 \% \mathrm{CI}(1.530-5.294))$ was a risk factor of one-year QoL, while tube extubation during the first 24 hours $(\mathrm{OR}=0.330,95 \% \mathrm{CI}(0.110-0.989))$ was a protective factor (Table 3 ).

\section{Discussion}

This study showed that short- and long-term outcomes between patients with pulmonary-sepsis and abdominalsepsis vary greatly. Our findings suggest that patients with pulmonary-sepsis were more prone to fungal infection, acute renal failure requiring CRRT, prolonged mechanical ventilation, longer ICU and hospital stays, and higher in-hospital and one-year mortality than the abdominal-sepsis group. In addition, the pulmonary-sepsis cohort had worse QoL indicators after hospital discharge. To our knowledge, our follow-up study was one of the few researches to investigate the clinical difference of the most frequently identified sepsis source, including short-term and long-term mortality, together with QoL.

Our study found that age, septic shock, acute renal failure, fungal infection, anion gap, and pulmonary infection were potential risk factors for increased one-year mortality. It is not surprising that older age positively correlates with higher long-term mortality. Septic shock is the most severe stage of sepsis and long-term outcome of septic shock was poor. Nesseler et al. [27] reported that 6-month mortality of septic shock was $45 \%$. Harris et al. [28] found that critically ill patients with acute kidney injury had higher one-year mortality, and it is reasonable to speculate that there was higher one-year mortality in patients with acute renal failure. Fungal infection usually occurs in patients with immunosuppression and was associated with increased hospital mortality [29]. Previous researches have shown that anion gap increases in $72 \%$ of critically ill patients, and elevated AG has been found to be associated with mortality in serious diseases, including critical illness [30-35].

The research revealed that pulmonary infection was associated with increased short-term and long-term mortality which was in accordance with previous studies. Mansur et al.'s study [36] reported a higher 90-day mortality in pulmonary-sepsis than abdominal-sepsis. Kim et al. [18] reported significantly higher $28 \mathrm{~d}$ mortality of pneumonia (41\%) than non-pulmonary-sepsis (30\%), and pneumonia was demonstrated to be a risk factor for 28-day mortality. In our study cohort, we found that pulmonary-sepsis patients were much older and had higher APACHE II, SOFA score, and Charlson Comorbidity Index. Comorbidities and laboratory parameters on admission of the sepsis cohort were shown in Table 1. Consistent with our study, the $\mathrm{PAO}_{2} / \mathrm{FiO}_{2}$ and $\mathrm{PaO}_{2}$ of pulmonary-sepsis patients were worse than other sepsis source patients and previous research had already validated $\mathrm{PaO}_{2} / \mathrm{FiO}_{2}$ as a biomarker for prognosis of sepsis such as mortality [18]. What is more, patients in the pulmonary-sepsis cohort were significantly older and had a higher rate of renal failure, thus explaining their higher APCHE II scores. The SOFA score of pulmonary-sepsis was apparently higher than that of the abdominal group $(p=0.003)$; however, this difference disappeared when comparing the nonpulmonary SOFA scores $(p=0.125)$; that is, the difference of SOFA scores between groups was primarily caused by the pulmonary component which can be explained by pneumonia. Pneumonia patients had a greater probability to have chronic pulmonary disease $(32 \%$ and 
TABLE 3: Univariate and multivariate regression analysis for risk factors of one-year mortality and quality of life.

\begin{tabular}{|c|c|c|c|c|}
\hline Predictors & OR $(95 \% \mathrm{CI})$ & $p^{\mathrm{a}}$ & OR $(95 \% \mathrm{CI})$ & $p^{\mathrm{b}}$ \\
\hline \multicolumn{5}{|c|}{ A: one-year mortality } \\
\hline Age & $1.036(1.017-1.043)$ & $0.000^{* *}$ & $1.025(1.011-1.039)$ & $0.001^{* *}$ \\
\hline APACHE II & $1.097(1.067-1.129)$ & $0.000^{* *}$ & - & - \\
\hline SOFA & $1.159(1.102-1.219)$ & $0.000^{* *}$ & - & - \\
\hline Charlson Comorbidity index & $1.068(1.012-1.126)$ & $0.016^{*}$ & - & - \\
\hline Malignancy & $1.770(1.034-3.030)$ & $0.045^{*}$ & - & - \\
\hline Septic shock & $2.327(1.549-3.495)$ & $0.000^{* *}$ & $2.533(1.525-5.568)$ & $0.000^{* *}$ \\
\hline Fungus infection & $2.213(1.424-3.167)$ & $0.000^{* *}$ & $1.846(1.160-2.938)$ & $0.010^{*}$ \\
\hline Acute renal failure & $3.854(2.152-6.904)$ & $0.000^{* *}$ & $2.914(1.525-5.568)$ & $0.001^{* *}$ \\
\hline Cystatin c & $1.453(1.193-1.768)$ & $0.000^{* *}$ & - & - \\
\hline Anion gap & $1.071(1.031-1.113)$ & $0.000^{* *}$ & $1.070(1.025-1.117)$ & $0.002^{* *}$ \\
\hline \multirow[t]{2}{*}{ Pulmonary infection $^{c}$} & $2.243(1.465-3.436)$ & $0.000^{* *}$ & $2.547(1.513-4.288)$ & $0.000^{* *}$ \\
\hline & \multicolumn{3}{|c|}{ B: one-year QoL } & \\
\hline APACHE II & $1.048(1.008-1.088)$ & $0.017^{*}$ & - & - \\
\hline Chronic heart failure & $6.217(1.343-28.786)$ & $0.019^{*}$ & - & - \\
\hline Pulmonary infection $^{c}$ & $2.939(1.621-5.329)$ & $0.000^{* *}$ & $2.846(1.530-5.294)$ & $0.004^{* *}$ \\
\hline Tracheal extubation in $24 \mathrm{~h}^{\mathrm{d}}$ & $0.231(0.083-0.645)$ & $0.005^{* *}$ & $0.330(0.110-0.989)$ & $0.048^{*}$ \\
\hline Mechanical ventilation days & $1.036(1.009-1.064)$ & $0.008^{* *}$ & - & - \\
\hline
\end{tabular}

A: $n=435$. Variables eliminated from backward selection.

B: $n=209$. Variables eliminated from backward selection.

${ }^{\mathrm{a}}$ Results of univariate analysis.

${ }^{\mathrm{b}}$ Results of multivariate analysis.

${ }^{c}$ Pulmonary infection was defined as pulmonary infection identified during the whole ICU stay period.

${ }^{\mathrm{d}} n=188$; patients without mechanical ventilation were excluded.

${ }^{*} p<0.05$. ${ }^{* *} p<0.01$.

TABLE 4: Baseline characteristics of sepsis survivors with good/poor one-year QoL.

\begin{tabular}{|c|c|c|c|}
\hline Variables & $\begin{array}{c}\text { Good QoL } \\
n=106\end{array}$ & $\begin{array}{c}\text { Poor QoL } \\
n=103\end{array}$ & $p$ \\
\hline Age, mean (SD) & $54.6(16.7)$ & $58.8(16.4)$ & 0.066 \\
\hline Male sex, $n \%$ & $80(75.5)$ & $63(61.2)$ & $0.037^{*}$ \\
\hline APACHE II, mean (SD) & $18.1(7.0)$ & $20.6(7.6)$ & $0.016^{*}$ \\
\hline SOFA, mean (SD) & $7.2(3.7)$ & $7.9(4.3)$ & 0.245 \\
\hline Charlson Comorbidity Index & $2(0,3)$ & $3(1,4)$ & $0.021^{*}$ \\
\hline Septic shock, $n \%$ & $60(56.6)$ & $57(55.3)$ & 0.890 \\
\hline Chronic heart failure, $n \%$ & $2(1.9)$ & $11(10.7)$ & $0.010^{*}$ \\
\hline Pulmonary infection ${ }^{\mathrm{a}}, n \%$ & $50(47.2)$ & $79(76.7)$ & $0.000^{* *}$ \\
\hline Pulmonary-sepsis, $n \%$ & $38(25.8)$ & $59(57.3)$ & $0.002^{* *}$ \\
\hline \multicolumn{4}{|l|}{ ICU treatment within $24 \mathrm{~h}, n \%$} \\
\hline IPPV & $88(83.0)$ & $87(84.5)$ & 0.852 \\
\hline NPPV & $3(2.8)$ & $11(10.7)$ & $0.028^{*}$ \\
\hline Tube extubation $^{\mathrm{b}}$ & $18(20.0)$ & $5(5.1)$ & $0.003^{* *}$ \\
\hline Vasopressor & $28(26.4)$ & $34(33.0)$ & 0.364 \\
\hline $\mathrm{MV}, \mathrm{d}$ & $6(22-12)$ & $8(4-17)$ & $0.003^{* *}$ \\
\hline ICU LOS, d & $11(6-19)$ & $15(7-27)$ & $0.016^{*}$ \\
\hline Hospital LOS, d & $26.5(16.8-42.3)$ & $31(20-58)$ & $0.034^{*}$ \\
\hline
\end{tabular}

Quantitative data was presented as median (IQR), and qualitative data was presented as $n$ (\%) except otherwise indicated. SD, standard deviation; IPPV, invasive ventilation; NPPV, noninvasive ventilation; MV, mechanical ventilation; ICU LOS, length of ICU stay; hospital LOS, length of hospital stay;

${ }^{a}$ Pulmonary infection was defined as pulmonary infection identified during the whole ICU stay period.

${ }^{\mathrm{b}} N=188$; patients without mechanical ventilation were excluded.

${ }^{*} p<0.05 .{ }^{* *} p<0.01$. 
$11.7 \%, p=0.000)$. COPD was the most common chronic pulmonary disease and the quality of life for patients with COPD was apparently impaired [37]. Greater portion of patients with cardiovascular disease, cerebrovascular disease, chronic pulmonary disease, and chronic kidney disease in the pulmonary-sepsis group also contributed to high longterm mortality [38-41]. In order to eliminate the impact of older age and age-associated diseases on the pulmonarysepsis cohort, an age-matched cohort analysis was conducted. Survival analysis of both the unmatched and the matched cohorts showed greater mortality in the pulmonary-sepsis group (Figures 2(a) and 2(b)).

Quality of life for sepsis was impaired [27, 42]. Patients with poor QoL were much older, had higher APACHE II, SOFA, and Charlson Comorbidity Index, and had prolonged mechanical ventilation days and ICU and hospital LOS (Table 4). Chronic heart failure was also found more commonly in the poor QoL group. A total of $57.3 \%$ of the 103 survivors in the poor QoL group were diagnosed with pulmonary-sepsis when admitted to the ICU and 76.7\% of survivors with poor QoL suffered pulmonary infection in ICU (Table 4). In accordance with data shown in Table 1, pulmonary-sepsis cases had older age, higher APACHE II and SOFA score, and greater comorbidity burden (Table 1, Figure 2). Patients with tube weaning in the first 24 hours had better QoL, since these patients tended to be less serious, had less need for mechanical ventilation, and could soon recover from the sepsis attack. Pulmonary infection was already confirmed to be a risk factor for $28 \mathrm{~d}$ mortality [18]. Our study was the first to confirm its role in decreased QoL.

There were several limitations in our study. Firstly, this follow-up study was a single-center study conducted in a teaching hospital. This study design would result in lack of representativeness. Patients admitted to our hospital appeared to be much more serious, and they were much older and had more complications than patients admitted to ICUs of other hospitals, resulting in an overestimation of mortality. Moreover, a majority of patients were transferred from other hospitals and patients fulfilling the sepsis criteria at the onset of disease might fail to be diagnosed as having sepsis. These could all lead to selection bias. Secondly, the evaluation of GCS was inaccurate due to the use of sedation and approximately half of the cohort did not have a measurement of lactate during the 24 hours. Thirdly, Tibetan patients who could not speak Mandarin were excluded for language barrier, increasing the rate of patients lost to followup. Multicenter studies with larger samples were needed to confirm the study results.

\section{Conclusions}

Patients diagnosed with sepsis show ongoing mortality after the sepsis episode, with only $63.9 \%$ surviving one year after ICU discharge. Pulmonary-sepsis had worse short-term and long-term outcomes, including ICU/hospital mortality, oneyear mortality, and one-year quality of life. Pulmonary infection is a risk factor for one-year mortality and is associated with decreased health-related quality of life.

\section{Competing Interests}

The authors declare that there is no conflict of interests regarding the publication of this paper.

\section{Acknowledgments}

The authors are grateful to Chao Jiang (Chongqing University, China) for designing the figures for the manuscript.

\section{References}

[1] G. S. Martin, D. M. Mannino, S. Eaton, and M. Moss, "The epidemiology of sepsis in the United States from 1979 through 2000," The New England Journal of Medicine, vol. 348, no. 16, pp. 1546-1554, 2003.

[2] V. Y. Dombrovskiy, A. A. Martin, J. Sunderram, and H. L. Paz, "Rapid increase in hospitalization and mortality rates for severe sepsis in the United States: a trend analysis from 1993 to 2003," Critical Care Medicine, vol. 35, no. 5, pp. 1244-1250, 2007.

[3] D. F. Gaieski, J. M. Edwards, M. J. Kallan, and B. G. Carr, "Benchmarking the incidence and mortality of severe sepsis in the united states," Critical Care Medicine, vol. 41, no. 5, pp. 1167$1174,2013$.

[4] J. Zhou, C. Qian, M. Zhao et al., "Epidemiology and outcome of severe sepsis and septic shock in intensive care units in Mainland China," PLoS ONE, vol. 9, no. 9, Article ID e107181, 2014.

[5] M. M. Levy, R. P. Dellinger, S. R. Townsend et al., "The surviving sepsis campaign: results of an international guideline-based performance improvement program targeting severe sepsis," Intensive Care Medicine, vol. 36, no. 2, pp. 222-231, 2010.

[6] O. Liesenfeld, L. Lehman, K. Hunfeld, and G. Kost, "Molecular diagnosis of sepsis: new aspects and recent developments," European Journal of Microbiology and Immunology, vol. 4, no. 1, pp. 1-25, 2014.

[7] D. K. Heyland, W. Hopman, H. Coo, J. Tranmer, and M. A. McColl, "Long-term health-related quality of life in survivors of sepsis. Short Form 36: a valid and reliable measure of healthrelated quality of life," Critical Care Medicine, vol. 28, no. 11, pp. 3599-3605, 2000.

[8] K. Reinhart, R. Daniels, N. Kissoon, J. O’Brien, F. R. Machado, and E. Jimenez, "The burden of sepsis-a call to action in support of World Sepsis Day 2013," Journal of Critical Care, vol. 28, no. 4, pp. 526-528, 2013.

[9] T. Götz, A. Günther, O. W. Witte, F. M. Brunkhorst, G. Seidel, and F. Hamzei, "Long-term sequelae of severe sepsis: cognitive impairment and structural brain alterations-an MRI study (LossCog MRI)," BMC Neurology, vol. 14, no. 1, article 145, 2014.

[10] C. Alberti, C. Brun-Buisson, S. V. Goodman et al., "Influence of systemic inflammatory response syndrome and sepsis on outcome of critically III infected patients," American Journal of Respiratory and Critical Care Medicine, vol. 168, no. 1, pp. 77-84, 2003.

[11] The EuroQol Group, "EuroQol—a new facility for the measurement of health-related quality of life," Health Policy, vol. 16, no. 3, pp. 199-208, 1990.

[12] C. Granja, C. Dias, A. Costa-Pereira, and A. Sarmento, "Quality of life of survivors from severe sepsis and septic shock may be similar to that of others who survive critical illness," Critical Care, vol. 8, no. 2, pp. R91-R98, 2004. 
[13] D. C. Angus and J. Carlet, "Surviving intensive care: a report from the 2002 Brussels Roundtable," Intensive Care Medicine, vol. 29, no. 3, pp. 368-377, 2003.

[14] A. Tsuchiya, S. Ikeda, N. Ikegami et al., "Estimating an EQ-5D population value set: the case of Japan," Health Economics, vol. 11, no. 4, pp. 341-353, 2002.

[15] M. A. Puskarich, S. Trzeciak, N. I. Shapiro, A. C. Heffner, J. A. Kline, and A. E. Jones, "Outcomes of patients undergoing early sepsis resuscitation for cryptic shock compared with overt shock," Resuscitation, vol. 82, no. 10, pp. 1289-1293, 2011.

[16] M. D. Howell, M. Donnino, P. Clardy, D. Talmor, and N. I. Shapiro, "Occult hypoperfusion and mortality in patients with suspected infection," Intensive Care Medicine, vol. 33, no. 11, pp. 1892-1899, 2007.

[17] S. Trzeciak, R. P. Dellinger, M. E. Chansky et al., "Serum lactate as a predictor of mortality in patients with infection," Intensive Care Medicine, vol. 33, no. 6, pp. 970-977, 2007.

[18] W.-Y. Kim, Y.-J. Lee, S. Yeon Lim et al., "Clinical characteristics and prognosis of pneumonia and sepsis: Multicenter Study," Minerva Anestesiologica, vol. 79, no. 12, pp. 1356-1365, 2013.

[19] S. Finfer, R. Bellomo, J. Lipman, C. French, G. Dobb, and J. Myburgh, "Adult-population incidence of severe sepsis in Australian and New Zealand intensive care units," Intensive Care Medicine, vol. 30, no. 4, pp. 589-596, 2004.

[20] J.-L. Vincent, J. Rello, J. Marshall et al., "International study of the prevalence and outcomes of infection in intensive care units," The Journal of the American Medical Association, vol. 302, no. 21, pp. 2323-2329, 2009.

[21] V. M. Ranieri, B. T. Thompson, P. S. Barie et al., "Drotrecogin alfa (activated) in adults with septic shock," The New England Journal of Medicine, vol. 366, no. 22, pp. 2055-2064, 2012.

[22] J. A. Russell, "Management of sepsis," The New England Journal of Medicine, vol. 355, no. 16, pp. 1699-1713, 2006.

[23] R. C. Bone, R. A. Balk, F. B. Cerra et al., "Definitions for sepsis and organ failure and guidelines for the use of innovative therapies in sepsis. The ACCP/SCCM Consensus Conference Committee. American College of Chest Physicians/Society of Critical Care Medicine," Chest, vol. 101, no. 6, pp. 1644-1655, 1992.

[24] American Thoracic Society and Infectious Diseases Society of America, "Guidelines for the management of adults with hospital-acquired, ventilator-associated, and healthcareassociated pneumonia," American Journal of Respiratory and Critical Care Medicine, vol. 171, no. 4, pp. 388-416, 2005.

[25] W. A. Knaus, E. A. Draper, D. P. Wagner, and J. E. Zimmerman, "APACHE II: a severity of disease classification system," Critical Care Medicine, vol. 13, no. 10, pp. 818-829, 1985.

[26] J.-L. Vincent, A. de Mendonça, F. Cantraine et al., "Use of the SOFA score to assess the incidence of organ dysfunction/failure in intensive care units: results of a multicenter, prospective study. Working group on 'sepsis-related problems' of the European Society of Intensive Care Medicine," Critical Care Medicine, vol. 26, no. 11, pp. 1793-1800, 1998.

[27] N. Nesseler, A. Defontaine, Y. Launey, J. Morcet, Y. Mallédant, and P. Seguin, "Long-term mortality and quality of life after septic shock: A Follow-Up Observational Study," Intensive Care Medicine, vol. 39, no. 5, pp. 881-888, 2013.

[28] D. G. Harris, M. P. McCrone, G. Koo et al., "Epidemiology and outcomes of acute kidney injury in critically ill surgical patients," Journal of Critical Care, vol. 30, no. 1, pp. 102-106, 2015.
[29] C. Lichtenstern, C. Herold, M. Mieth et al., "Relevance of Candida and other mycoses for morbidity and mortality in severe sepsis and septic shock due to peritonitis," Mycoses, vol. 58, no. 7, pp. 399-407, 2015.

[30] G. Domínguez-Cherit and S. A. Ñamendys-Silva, "Changes in the anion gap: a novel marker of outcome in critically ill patients. Back to the basis," Critical Care Medicine, vol. 41, no. 1, pp. 336-337, 2013.

[31] M. K. Abramowitz, T. H. Hostetter, and M. L. Melamed, “The serum anion gap is altered in early kidney disease and associates with mortality," Kidney International, vol. 82, no. 6, pp. 701-709, 2012.

[32] J. J. Leskovan, C. F. Justiniano, J. A. Bach et al., "Anion gap as a predictor of trauma outcomes in the older trauma population: correlations with injury severity and mortality," The American Surgeon, vol. 79, no. 11, pp. 1203-1206, 2013.

[33] C.-M. Zheng, W.-C. Liu, J.-Q. Zheng et al., "Metabolic acidosis and strong ion gap in critically ill patients with acute kidney injury," BioMed Research International, vol. 2014, Article ID 819528, 8 pages, 2014.

[34] S. H. Lee, S. Park, J. W. Lee et al., "The anion gap is a predictive clinical marker for death in patients with acute pesticide intoxication," Journal of Korean Medical Science, vol. 31, no. 7, pp. 1150-1159, 2016.

[35] M. S. Lipnick, A. B. Braun, J. T.-W. Cheung, F. K. Gibbons, and K. B. Christopher, "The difference between critical care initiation anion gap and prehospital admission anion gap is predictive of mortality in critical illness," Critical Care Medicine, vol. 41, no. 1, pp. 49-59, 2013.

[36] A. Mansur, Y. Klee, A. F. Popov et al., "Primary bacteraemia is associated with a higher mortality risk compared with pulmonary and intra-abdominal infections in patients with sepsis: a prospective observational cohort study," BMJ Open, vol. 5, no. 1, Article ID e006616, 2015.

[37] S. Spencer, P. M. A. Calverley, P. S. Burge, and P. W. Jones, "Impact of preventing exacerbations on deterioration of health status in COPD," The European Respiratory Journal, vol. 23, no. 5, pp. 698-702, 2004.

[38] Y. R. Chin, I. S. Lee, and H. Y. Lee, "Effects of hypertension, diabetes, and/or cardiovascular disease on health-related quality of life in elderly Korean individuals: a population-based crosssectional survey," Asian Nursing Research, vol. 8, no. 4, pp. 267273, 2014.

[39] J. Persson, L. Holmegaard, I. Karlberg et al., "Spouses of stroke survivors report reduced health-related quality of life even in long-term follow-up: results from Sahlgrenska Academy study on ischemic stroke," Stroke, vol. 46, no. 9, pp. 2584-2590, 2015.

[40] J. Sundh, G. Johansson, K. Larsson et al., "Comorbidity and health-related quality of life in patients with severe chronic obstructive pulmonary disease attending Swedish secondary care units," International Journal of Chronic Obstructive Pulmonary Disease, vol. 10, pp. 173-183, 2015.

[41] S. J. Lee, H. Son, and S. K. Shin, "Influence of frailty on healthrelated quality of life in pre-dialysis patients with chronic kidney disease in Korea: a cross-sectional study," Health and Quality of Life Outcomes, vol. 13, article 70, 2015.

[42] B. H. Cuthbertson, A. Elders, S. Hall et al., "Mortality and quality of life in the five years after severe sepsis," Critical Care, vol. 17, no. 2, article R70, 2013. 


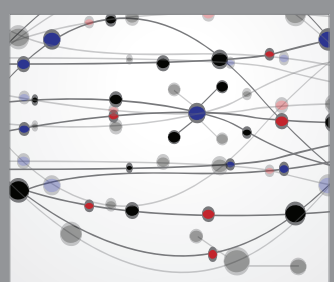

The Scientific World Journal
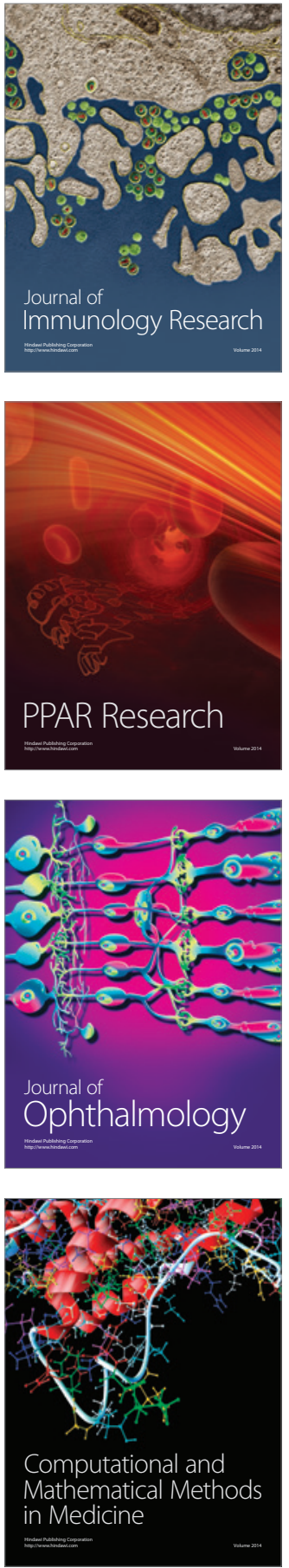

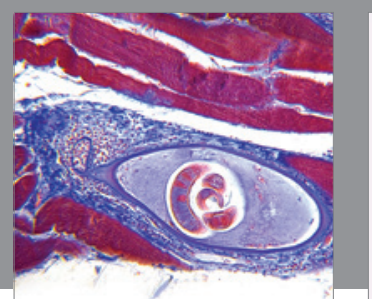

Gastroenterology Research and Practice

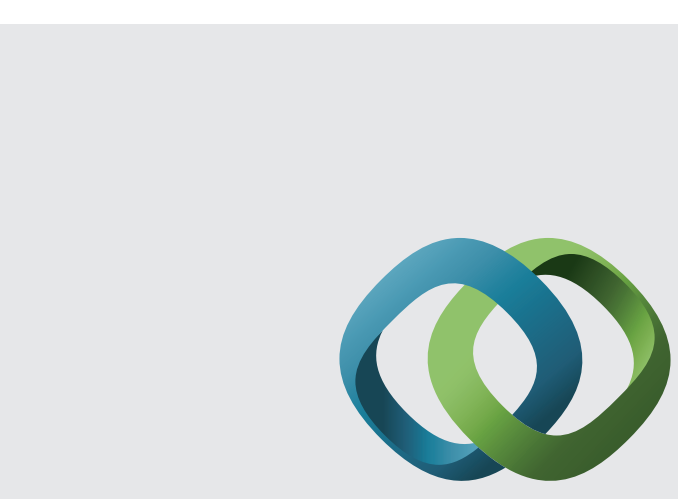

\section{Hindawi}

Submit your manuscripts at

http://www.hindawi.com
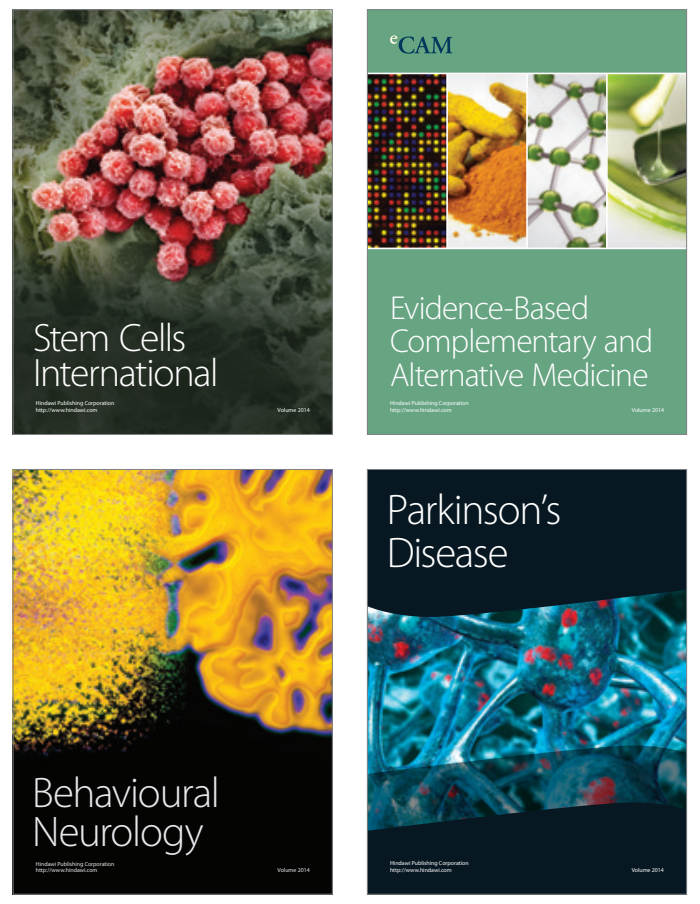
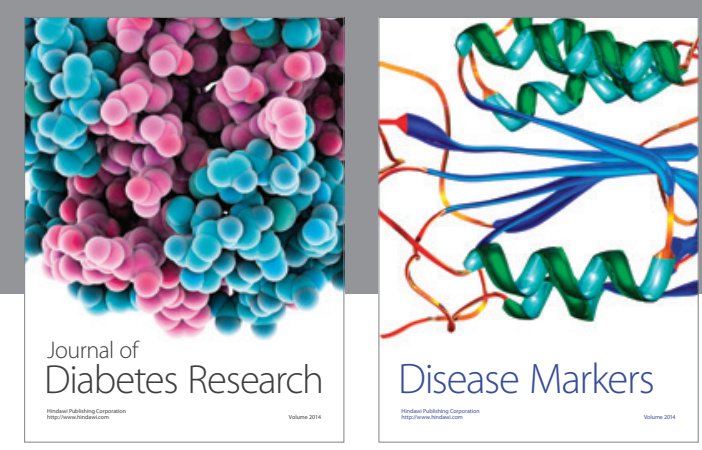

Disease Markers
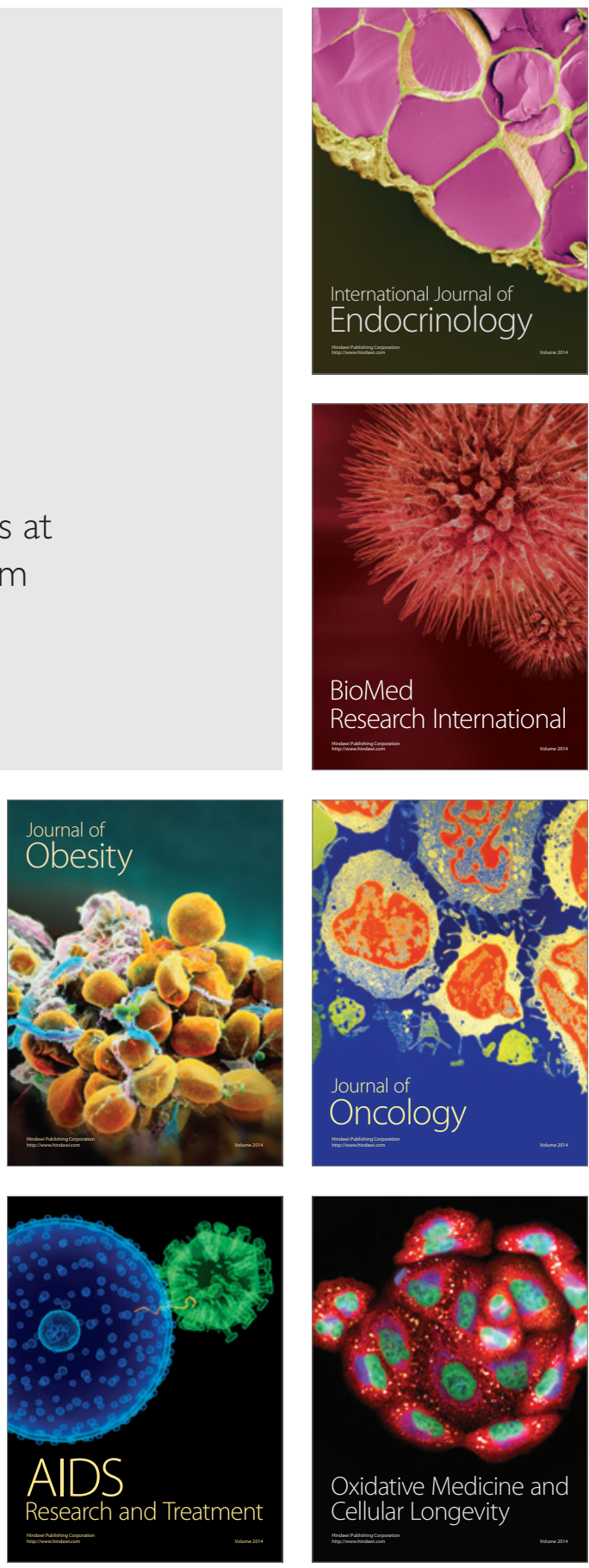